\section{¿Es eficiente la protección anti-radiación otorgada por gorros de pabellón de tungsteno-bismuto en cardiología intervencionista?}

\author{
SERGIO RAMOS-AVASOLA ${ }^{1,2, a, d}$, NATALIA DÍAZ ${ }^{1, g}$, \\ REYNALDO ROLDÁN ${ }^{1, g}$, JORGE GAMARRA ${ }^{3, b, e}$, MÓNICA CATALÁN ${ }^{4, c, f}$

\section{Radiation protection provided interventional cardiology} \\ by tungsten bismuth caps during
}

Background: The effectiveness against radiation of tungsten bismuth caps, used in interventional cardiology is not well known. Aim: To determine the degree of radiation protection conferred by these caps in real work conditions. Material and Methods: We compared the gross electric charges received at brain lobe levels by three occupationally exposed professionals who participated in 22 consecutive procedures, inside and outside of the tungsten bismuth cap. Results: The median electric charges outside and inside the cap were 3.71 (range 1.46-5.62) and 2.2 (range 1.29-3.93) $n C$, which correspond to a $40 \%$ radiation attenuation. However, the protection was heterogeneous. Conclusions: Tungsten bismuth caps provide an adequate attenuation, but its degree is heterogeneous.

(Rev Med Chile 2016; 144: 837-843)

Key words: Endovascular Procedures; Occupational Exposure; Occupational Health; Radiation Protection.
'Escuela de Tecnología Médica. Facultad de Ciencias de la Salud. Universidad Viña del Mar. Viña del Mar, Chile.

${ }^{2}$ Unidad de Hemodinamia Hospital Dr. Gustavo Fricke, Viña del Mar, Chile. ${ }^{3}$ Comisión Chilena de Energía Nuclear, Santiago, Chile.

${ }^{4}$ Instituto de Estadística, Facultad de Ciencias, Universidad de Valparaíso,

Valparaíso, Chile.

aTecnólogo Médico.

bFísico.

'Estadístico.

¿Magíster en Epidemiología.

'Magíster en Biofísica Médica.

fDoctor en Estadística.

${ }^{9}$ Alumno tesista de la Escuela de Tecnología Médica de la Universidad Viña del Mar. Viña del Mar, Chile.

Fuentes de financiamiento: Esta investigación fue financiada por la Empresa Cardiotec y el laboratorio $X$-Ray, quienes aportaron el costo de los cristales TLDs, pero no intervinieron en el diseño, recolección, análisis, preparación, revisión o aprobación del manuscrito.

Recibido el 31 de diciembre de 2015 , aceptado el 5 de julio de 2016 .

Correspondencia a:

Sergio Ramos Avasola

Universidad Viña del Mar, Viña del Mar, Chile.

Facultad de Ciencias de La Salud. Escuela de Tecnología Médica. Agua Santa 7055, sector Rodelillo, Viña del Mar. Chile.

sergioramosavasola@gmail.com
E s un hecho reconocido que los elementos de radioprotección reducen gran parte de la radiación que recibe el profesional ocupacionalmente expuesto (POE), como el caso del delantal plomado, el cual atenúa la radiación entre 90 y $97 \%$, dependiendo del contenido en plomo del mismo ${ }^{1}$. Las medidas de radioprotección se enfocan principalmente en proteger los órganos más radiosensibles como el cristalino, gónadas y tiroides. La protección de otras partes del cuerpo ha sido relegada a un segundo plano. Un factor responsable de esto es la desinformación sobre los efectos a nivel de cerebro, ya que este órgano es considerado de baja radiosensibilidad debido a la protección que le confiere la bóveda craneal. Por otro lado, los escasos estudios ${ }^{2-4}$ publicados sobre la exposición a radiación a nivel del cerebro se han enfocado en el cardiólogo intervencionista, sin considerar los otros profesionales que los acompañan; en uno de estos estudios, se describió a médicos intervencionistas que han manifestado lesiones tumorales, preferentemente en el hemisferio cerebral izquierdo ${ }^{2}$. Aun cuando no se demostró que la exposición a radiación ionizante en forma constante sea la causa de esas lesiones, tampoco se descartó dicha posibilidad. En cuanto a la efectividad de los gorros con materiales radioprotectores $(\mathrm{Pb} 0,5$; sulfato de bario-óxido de bismuto equivalente a $0,5 \mathrm{~mm}$ de $\mathrm{Pb}$ ), estos demostraron ser efectivos en reducir la radiación ${ }^{5-7}$.

Los cardiólogos intervencionistas trabajan varias horas diariamente y por muchos años, existiendo una exposición acumulada significativa de radiación ionizante ${ }^{3,8}$. En dos publicaciones ${ }^{9,10}$ se 
evaluó los efectos en el ADN somático provocados por la exposición a radiación ionizante en los cardiólogos intervencionistas, llegando a la conclusión que los daños citogenéticos registraron un daño cromosómico superior, en comparación a los cardiólogos clínicos que trabajan fuera del laboratorio de hemodinamia. En otros dos estudios ${ }^{2,3}$, se describieron 31 casos de tumores cerebrales en cardiólogos intervencionistas, electrofisiólogos y radiólogos intervencionistas. En estos artículos se destacó que el lado izquierdo de la cabeza fue el más expuesto a la radiación ionizante, siendo dos veces más en comparación que el lado derecho. La exposición anual de la cabeza de estos especialistas estuvo en el rango de 20 a $30 \mathrm{mSv}$ por año, alrededor de 10 veces más que toda la exposición del cuerpo $^{2,3}$. Un quinto estudio ${ }^{4}$, un reporte de caso, un cardiólogo intervencionista evaluó la eficacia de un gorro plomado en conjunto con la pantalla protectora de vidrio plomado. Este estudio reveló que la utilización del gorro plomado disminuye las dosis de radiación en la cabeza.

Antiguamente se empleaban gorros plomados, fabricados con láminas de plomo de un peso aproximado de $1,14 \mathrm{~kg}$, lo que hacía que fueran incómodos de utilizar ${ }^{4}$, esto provocó que se dejaran de utilizar. Hoy en día, se han creado gorros protectores formados por delgadas láminas de distintas aleaciones de materiales, como tungsteno y bismuto, con el fin de reducir el peso, aumentar la flexibilidad y disminuir el tamaño del gorro protector, se sabe muy poco sobre la eficiencia de estos nuevos gorros protectores. Además, no existen estudios enfocados en diferenciar qué lóbulos cerebrales estarían más afectados por la radiación ionizante en el POE. Es preciso estudiar a cada POE en conjunto con el médico intervencionista, ya que en muchos casos los anestesistas, tecnólogos médicos y arsenaleros están presentes en hasta tres veces más procedimientos que los médicos.

El objetivo fundamental de este estudio es comprobar el grado de atenuación otorgado por los gorros de tungsteno-bismuto (GTB), tanto para el médico intervencionista, como en los otros profesionales de la salud que lo acompañan.

\section{Material y Métodos}

Este estudio fue aprobado por el Comité de Ética de la Universidad Viña del Mar, involucró a tres POE en una unidad de hemodinamia de la V Región de Chile, que estuvieron expuestos a una fuente de radiación ionizante secundaria generada en 22 procedimientos cardiológicos intervencionistas. El equipo utilizado fue un angiógrafo marca Siemens, modelo Axiom Artis Df (Múnich, Alemania), del año 2005, el cual se encuentra adscrito a un protocolo de mantención preventiva y reparativa cada 4 meses, equipado con un panel plano para estudios cardíacos. Al momento de la realización de cada procedimiento angiográfico se encontraban en el pabellón un cardiólogo intervencionista, un técnico paramédico, un tecnólogo médico y una enfermera, los cuales estaban distribuidos en el pabellón de acuerdo a la Figura 1. Todos ellos debidamente acreditados con cursos de radioprotección.

Al momento de los exámenes los POE utilizaron delantales plomados, protectores tiroideos y gafas plomadas, que son de uso personal y exclusivo, con una equivalencia de 0,$5 ; 0,5$ y $0,25 \mathrm{~mm} /$ $\mathrm{Pb}$ respectivamente. Adicionalmente se utilizó un GTB RADPAD ${ }^{\circledR} 9100$ No Brainer ${ }^{\circledR}$ (RADPAD, $\mathrm{KA}, \mathrm{USA}$ ) con una equivalencia de $0,125 \mathrm{~mm} / \mathrm{Pb}$. También se utilizó una pantalla de vidrio plomado suspendida del techo y un faldón plomado bajo la camilla, con una equivalencia de $0,5 \mathrm{~mm} / \mathrm{Pb}$. En cuanto a la disposición de los integrantes del POE dentro del pabellón, esta fue siempre la misma.

En relación a los parámetros dependientes del angiógrafo, la fluoroscopia fue pulsada con 15 pulsos/segundo, la cinecoronariografía se realizó con 15 imágenes/segundo y la colimación de las adquisiciones fue realizada de forma habitual. Respecto a las dosis ambientales de la unidad donde se realizó el experimento, esta fue de $0,2 \mathrm{mSv}$.

\section{Mediciones}

Las cargas eléctricas brutas medidas dentro y fuera del GTB fueron medidas en nanocoulombs (nC) con 16 cristales termoluminicentes marca Harshaw, modelo TLD-100 (Lif:Mg,Ti), (Massachusetts, USA) por persona, dispuestos dentro y fuera del GTB a la altura y posición de cada uno de los lóbulos cerebrales, como se puede apreciar en la Figura 2. Estos cristales tienen un rango de medición de 10 a 1.000.000 $\mu$ Sv y fueron proporcionados por el laboratorio X-Ray y posteriormente leídos en el Laboratorio de Radioactividad y Termoluminiscencia de la Pontificia Universidad 


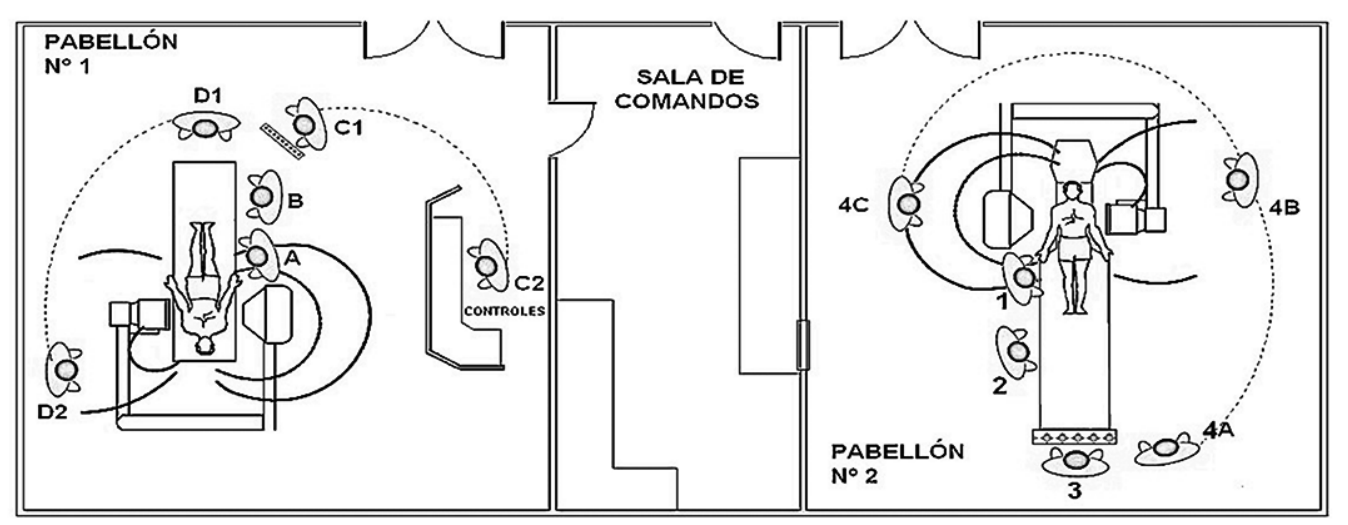

Figura 1. 1 = Médico intervencionista; 2 = Técnico paramédico; 3 = Tecnólogo médico; 4 = Enfermera (Imagen cedida gentilmente por Dr. Cristian Fernández Palomo. Tesis de pregrado "Detección y Medición de Radiación lonizante Secundaria Generada por un Equipo de Angiografía Coronaria" de la Escuela de Tecnología Médica, Universidad de Valparaíso, 2006).

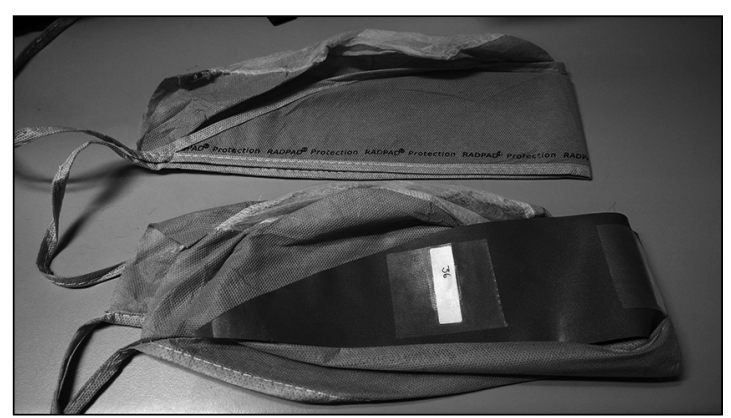

Figura 2. Disposición de un dosímetro colocado en la parte externa de la lámina de tungsteno-bismuto a nivel temporoparietal derecho. El segundo dosímetro queda oculto en la imagen, pero está a la misma altura por la parte interna de la lámina (Fuente: elaboración propia).

Católica de Santiago de Chile (LRT/PUC), laboratorio adscrito al Programa Nacional de Intercomparación, dependiente del Instituto de Salud Pública de Chile, se deja constancia que ambos laboratorios utilizan el mismo tipo de detector y de igual sensibilidad, además, previo a su utilización, los cristales fueron sometidos al riguroso protocolo de medición del laboratorio de dosimetría. Para prevenir el sesgo de medición no se le indicó al laboratorio de dosimetría la ubicación de los dosímetros en el pabellón de hemodinamia, por lo tanto fueron evaluadores ciegos.

Una vez instalados los cristales, estos fueron portados por los miembros del POE por un período de una semana, tras el cual fueron trasladados al LRT/PUC donde fueron medidos en un lector automático Harshaw TLD Modelo 3500. (ThermoFisher Scientific, Waltham, MA, USA).

El producto dosis-área (DAP, dose-area pro$d u c t)$ se define como el producto del área transversal del haz de rayos X y el promedio Kerma aire (energía cinética entregada a la materia) sobre la sección transversal y es ampliamente utilizado para evaluar la dosis del paciente en procedimientos radiográficos y fluoroscópicos, pero también puede ser usado para evaluar la magnitud de las dosis ocupacionales recibidas por el staff médico ${ }^{11}$. Las unidades en que se expresa este tipo de dosis son: $\mathrm{Cycm} 2, \mathrm{cCycm} 2, \mathrm{mCycm} 2$ y $\mu \mathrm{Cym} 2$, en esta investigación se utilizara el $\mu$ Cym. El DAP fue obtenido de la cámara de ionización integrada que tiene el equipo a la salida del tubo de rayos-X, que es calibrada periódicamente cada 6 meses.

\section{Análisis estadístico}

El análisis estadístico fue desarrollado utilizando un software estadístico (SPSS, versión 17. Cihicago, IL, USA). Los datos continuos (DAP, cargas eléctricas brutas) son expresados como la media \pm desviación estándar (SD) y los datos categóricos son expresados en porcentaje y su frecuencia absoluta. Dada la falta de normalidad, se utilizó la prueba de Wilcoxon para datos inde- 
pendientes cuando se comparó datos continuos. El nivel de significancia estadística utilizado fue de $\mathrm{p}<0,05$.

\section{Resultados}

El POE estuvo conformado por tres personas de: 67, 48 y 30 años (médico, tecnólogo médico y arsenalero, respectivamente), con una experiencia de: 32,16 y 4 años, respectivamente. Se realizaron 22 procedimientos endovasculares consecutivos que incluyeron: 1 implante de endoprótesis aórtica $\left(\mathrm{DAP}_{\text {acumulado }}=24.660,0 \mu \mathrm{Gym}^{2}\right), 12$ angiografías coronarias $\left(\mathrm{DAP}_{\text {acumulado }}=16.518,4 \mu \mathrm{Gym}^{2}\right)$ y 9 angioplastías coronarias $\left(\mathrm{DAP}_{\text {acumulado }}=27.138,6\right.$ $\left.\mu \mathrm{Gym}^{2}\right)$. En cuanto a la complejidad de las angioplastias abordadas, $22 \%$ correspondió a angioplastías de lesiones en bifurcación, 10\% a lesiones crónicamente ocluidas y el resto fueron angioplastías clasificadas como tipo A, B1 y B2 según la clasificación del Task Force ACC-AHA. El tiempo promedio de fluoroscopía empleado en los exámenes fue de 3,55 min.

Los niveles de carga eléctrica bruta acumulada recibida por los cristales termoluminiscentes portados por el POE a nivel de los lóbulos cerebrales son presentados en la Tabla 1 . Se aprecia que el GTB confirió un grado de atenuación de la radiación, que va desde $9,09 \%$ a $60,85 \%$ (lóbulo témporo-parietal derecho del médico intervencionista y el lóbulo témporo-parietal izquierdo del médico intervencionista, respectivamente). Interesante son los casos del tecnólogo médico y el arsenalero, donde la atenuación de la radiación observada se comportó a la inversa de lo esperado con -138,18\% y $-10,96 \%$ de atenuación, es decir, se registraron mayores cargas eléctricas dentro del gorro que fuera de este, en la zona del lóbulo occipital para el caso del tecnólogo médico y en el lóbulo témporo-parietal derecho para el arsenalero.

\section{Porcentaje de atenuación}

En términos absolutos, el valor de la mediana de la carga eléctrica bruta de los doce cristales dispuestos dentro del GTB de los tres sujetos estudiados fue de 2,20 $\mathrm{nC}$, rango $(1,29-3,93) \mathrm{y}$ el valor de la mediana de la carga eléctrica bruta de los doce cristales dispuestos fuera del GTB de los tres sujetos estudiados fue de $3,71 \mathrm{nC}$, rango $(1,46-5,62)$, lo que equivale a una atenuación de $40,2 \%$, diferencia que estadísticamente mostró ser significativa con un valor $\mathrm{p}=0,028$ (Test de Wilcoxon) (Figura 3).

Tabla 1. Lectura de Carga Bruta Promedio y porcentaje de atenuación proporcionado por los cristales termoluminiscentes (internos y externos)

\begin{tabular}{|c|c|c|c|}
\hline Estamento/lóbulo & CBP Externa (nC) & CBP Interna (nC) & $\%$ de atenuación \\
\hline \multicolumn{4}{|l|}{ Médico intervencionista } \\
\hline Frontal & 4,14 & 2,45 & 40,82 \\
\hline TP Derecho & 1,65 & 1,50 & 9,09 \\
\hline TP Izquierdo & 5,62 & 2,20 & 60,85 \\
\hline Occipital & 5,41 & 2,53 & 53,23 \\
\hline \multicolumn{4}{|l|}{ Tecnólogo Médico } \\
\hline Frontal & 3,88 & 1,53 & 60,57 \\
\hline TP Derecho & 3,71 & 2,91 & 21,56 \\
\hline TP Izquierdo & 3,21 & 2,65 & 17,45 \\
\hline Occipital & 1,65 & 3,93 & $-138,18$ \\
\hline \multicolumn{4}{|l|}{ Arsenalero } \\
\hline Frontal & 2,84 & 1,29 & 54,58 \\
\hline TP Derecho & 1,46 & 1,62 & $-10,96$ \\
\hline TP Izquierdo & 3,85 & 2,78 & 27,79 \\
\hline Occipital & 4,85 & 2,12 & 56,29 \\
\hline
\end{tabular}

CBP: carga bruta promedio. nC: nano Coulomb. 


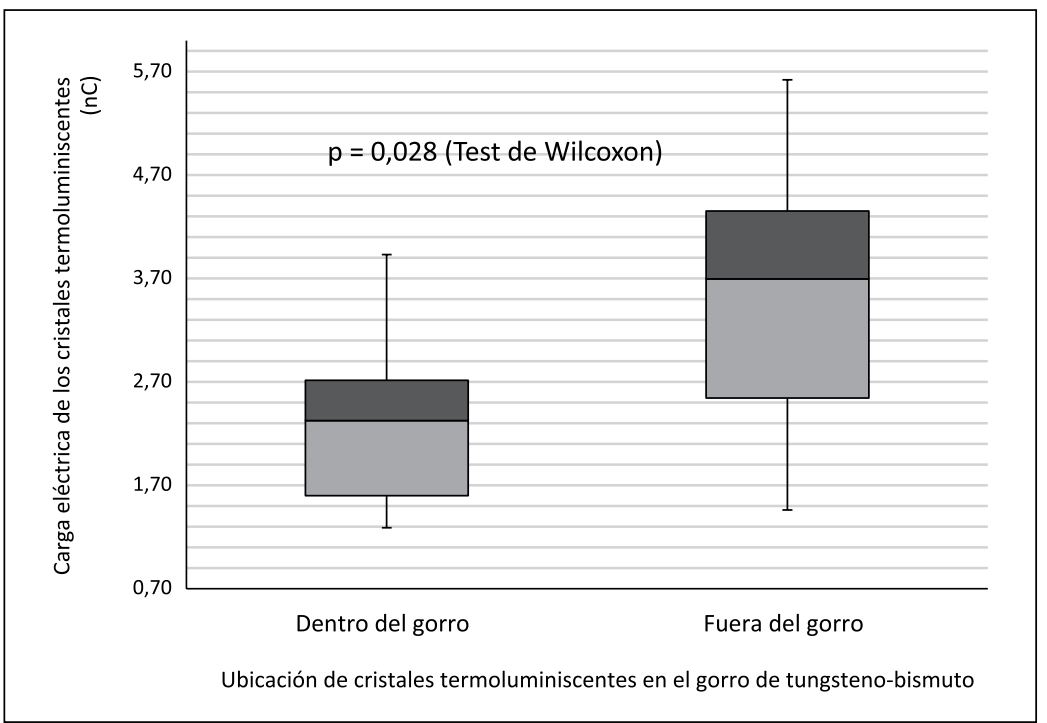

Figura 3. Carga eléctrica total registrada por los dosímetros fuera y dentro del blindaje.

\section{Discusión}

Con respecto a las características de la exposición a la que estuvieron sometidos el POE en este estudio, es decir, la emisión de radiación ionizante proporcionada por el equipo angiográfico utilizado en los procedimientos, se lograron condiciones experimentales acordes a las condiciones descritas en otros estudios ${ }^{12,13}$.

Las lecturas realizadas se comportaron de distinta forma según el profesional evaluado. La ubicación del POE en el pabellón de hemodinamia puede ser una razón por la cual no se logra una atenuación homogénea en todos los lóbulos cerebrales (Figura 4). El hecho que el tecnólogo médico, dada su ubicación habitual lejano a la fuente de rayos- $X$, no implica que no esté expuesto a dosis significativas de radiación, pues no tiene, como el médico, una barrera protectora plomada entre el paciente y él, además, habitualmente es el tipo de POE que más tiempo está expuesto, generalmente presente en $100 \%$ de los procedimientos, en cambio los otros POEs se alternan entre procedimientos, esto podría explicar los valores registrados en los cristales dispuestos en la zona de los lóbulos témporo-parietales derecho e izquierdo. El médico intervencionista se encuentra directamente protegido por un vidrio plomado, el cual atenúa la radiación secundaria proveniente del paciente; el arsenalero se encuentra ubicado

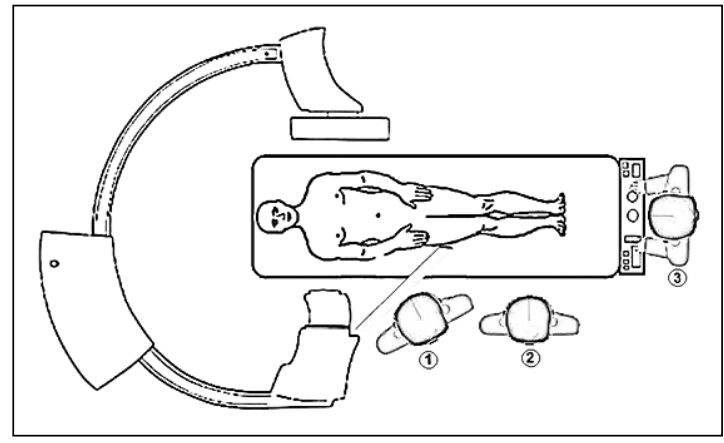

Figura 4. Representación de la ubicación de los profesionales dentro del pabellón de Hemodinamia observada desde arriba. 1) Médico intervencionista; 2) Arsenalero; 3) Tecnólogo médico (Fuente: elaboración propia).

cerca del médico, por lo que también recibe protección de este vidrio, protección que no es tan efectiva para el arsenalero pues, el médico es quien ubica este vidrio plomado en función de su protección, es así como en algunos momentos los haces de rayos-X primarios y secundarios logran impactar libremente y en forma oblicua el cerebro del arsenalero. Esto explicaría, posiblemente, por qué no se obtienen los resultados esperados considerando la distancia que existe desde la fuente o desde el paciente a cada estamento, en relación a resultados similares entregados por otro estudio ${ }^{12}$. Se debe tener en cuenta ciertas consideraciones ${ }^{14,15}$ 
al momento de valorar los cambios de distancia, ya que esto afecta las dosis ocupacionales, si bien las posiciones de los POEs durante estos exámenes son fijas, por lo tanto la distancia a la fuentes siempre es la misma, pero lo que cambia es la posición del tubo de rayos-X del angiógrafo, porque este cambia frecuentemente en función de la proyección que se realiza.

Es así como en el caso del médico intervencionista se confirmó una mayor exposición a la radiación en el lóbulo témporo-parietal izquierdo, lo cual previamente había sido ya descrito en otros estudios $^{2,3}$, el GTB logró atenuar la radiación en todas las zonas evaluadas, aunque en el lóbulo témporo-parietal derecho dicha atenuación es mínima (9,09\%), en cambio en el lóbulo témporo-parietal izquierdo fue máxima, con $60 \%$. Una probable explicación a este resultado se debería a que en su recorrido los haces de rayos-X desde la fuente a los dosímetros ubicados en el lado derecho es distinto a los dosímetros ubicados en el lado izquierdo, es así como para llegar los haces al dosímetro ubicado por fuera y en el lado derecho del cráneo deben atravesar adicionalmente la bóveda craneana.

La protección radiológica empleada por el POE en cardiología intervencionista posee rangos de equivalencia de plomo entre 0,25 y $0,5 \mathrm{~mm}$, como indica la Agencia Internacional de Energía Atómica, quienes declaran que para los profesionales de la salud, el uso del delantal plomado con una equivalencia de $0,5 \mathrm{~mm} / \mathrm{Pb}$, en conjunto con el escudo plomado, protectores de tiroides y lentes plomados, reduce la radiación en más de 95\%. Posiblemente, este fue un factor que pudo afectar los resultados de esta investigación, ya que se utilizó una equivalencia de $\mathrm{Pb}$ menor en el material radioprotector del gorro. Otro estudio que evaluó gafas plomadas, también obtuvo un bajo nivel de atenuación al emplear gafas con una equivalencia de $\mathrm{Pb}$ igual a $0,125^{12}$, en cambio los datos obtenidos por otros estudios ${ }^{4,14}$ realizados con blindaje de grosor adecuado lograron alcanzar una mejor respuesta de protección.

Con respecto a los valores registrados en el lóbulo occipital del tecnólogo médico y el lóbulo témporo-parietal derecho del arsenalero, se sospecha que el ángulo en que inciden los haces de radiación dispersa proveniente del paciente hacia la cabeza del POE (Figura 5), provoca que algunos de ellos atraviesan el cráneo directamente a la zona interna del GTB, para posteriormente atravesar la lámina de tungsteno bismuto y salir al exterior de la zona occipital, obteniéndose un porcentaje de atenuación inverso a lo esperado. La zona temporoparietal derecha del arsenalero presentó el mismo comportamiento, probablemente esto estaría dado por el ángulo en que inciden los haces de radiación en el cerebro del arsenalero.

Es recomendable, ante lo expuesto en este estudio, evaluar nuevos diseños de investigación con un mayor poder de causalidad y con nuevos modelos de gorros protectores que tomen en consideración en su diseño: la distancia del POE respecto a la fuente, el ángulo en el cual inciden los haces de radiación hacia el POE, el grosor de las láminas de tungsteno-bismuto y la posición en el pabellón de cada uno de los POE, lo que permitiría crear nuevos prototipos de gorros más eficientes (Figura 6), en el caso del tecnólogo médico, se podría utilizar un vidrio plomado y un gorro que abarque mayor zona de la cabeza (Figura 7).

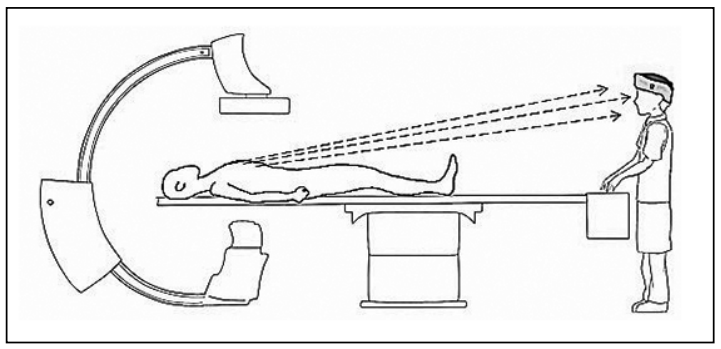

Figura 5. Representación de los distintos ángulos en los que puede ir la radiación dispersa hacia la cabeza del tecnólogo médico (Fuente: elaboración propia).

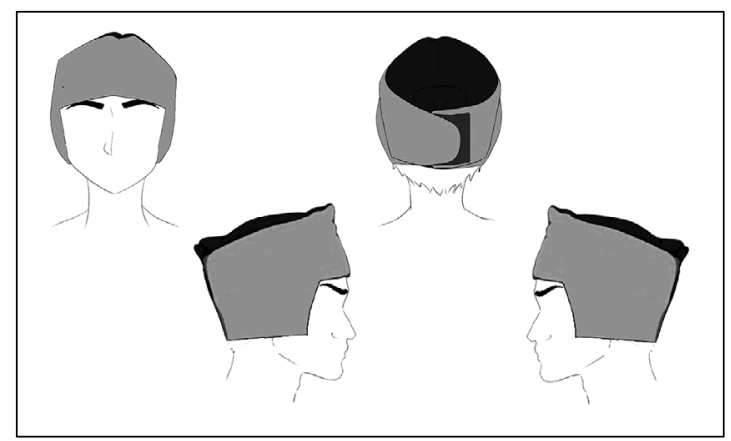

Figura 6. Prototipo de gorro radioprotector para médico y arsenalero con equivalencias de $\mathrm{Pb}$ diferenciales para el lado derecho e izquierdo del gorro (Fuente: elaboración propia). 


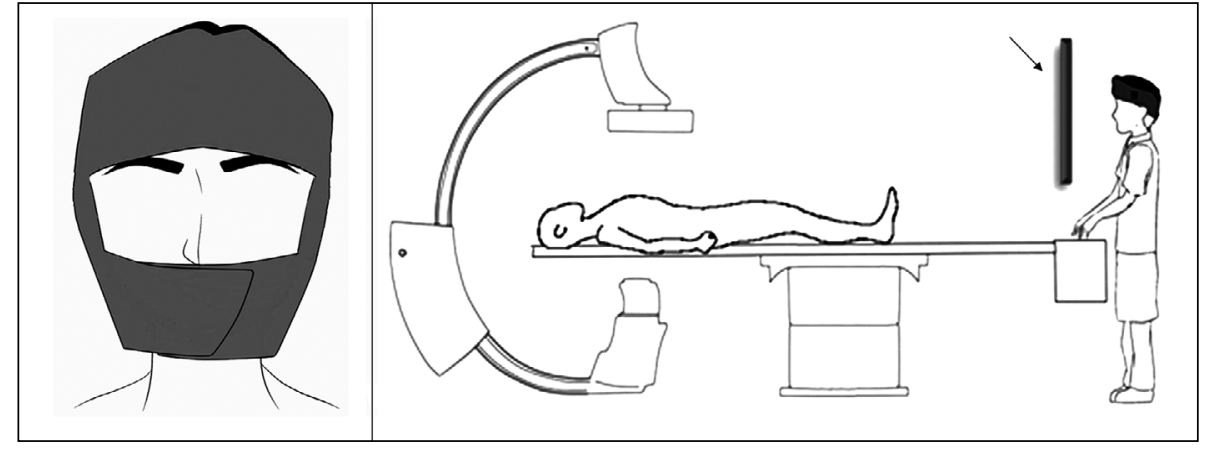

Figura 7. a) Prototipo de gorro radioprotector; b) Utilización de vidrio plomado como protección para el tecnólogo médico (Fuente: elaboración propia).
Agradecimientos: A la Empresa Cardiotec en la persona de la Srta. Andrea Vidal y al Laboratorio de Dosimetría X-Ray protección Radiológica en la persona del señor Derek Labbé por el valioso aporte de cristales TLDs, al Laboratorio de Dosimetría de la Pontificia Universidad Católica de Chile, a su personal: Dra. Paola Caprille, Claudia Morales y al Ingeniero Rubén Jerez, quienes dieron todas las facilidades para el análisis de las dosimetrías TLDs en su laboratorio.

\section{Referencias}

1. IAEA. Staff Radiation Protection [Internet]. [cited 2015 Sep 15]. Available from: https://rpop.iaea.org/RPOP/ RPoP/Content/InformationFor/HealthProfessionals/4_ InterventionalRadiology/fluoroscopy-operating-theatres/fluoroscopy-staff-protection.htm\#FULS-FAQ03

2. Roguin A, Goldstein J, Bar O, Goldstein JA. Brain and neck tumors among physicians performing interventional procedures. Am J Cardiol. Elsevier Inc 2013; 111 (9): 1368-72.

3. Roguin A, Goldstein J, Bar O. Brain tumours among interventional cardiologists: a cause for alarm? Report of four new cases from two cities and a review of the literature. EuroIntervention 2012; 7 (9): 1-4.

4. Karadag B, Ikitimur B, Durmaz E, Avci BK. Effectiveness of a lead cap in radiation protection of the head in the cardiac catheterisation laboratory. EuroIntervention 2013; 9: 754-6.

5. Uthoff H, Quesada R, Roberts JS, Baumann F, Schernthaner M, Zaremski L, et al. Radioprotective lightweight caps in the interventional cardiology setting: A randomised controlled trial (PROTECT). EuroIntervention 2015; 11 (1): 53-9.

6. Uthoff H, Peña C, West J, Contreras F, Benenati JF, Katzen BT. Evaluation of novel disposable, light-weight radiation protection devices in an interventional radiology setting: A randomized controlled trial. Am J Roentgenol 2013; 200 (4): 915-20.

7. Kuon E, Birkel J, Schmitt MBD. Radiation exposure benefit of a lead cap in invasive cardiology 2003; 1205-10.

8. Efstathopoulos EP, Pantos I, Andreou M, Gkatzis A, Carinou E, Koukorava C, et al. Occupational radiation doses to the extremities and the eyes in interventional radiology and cardiology procedures. Br J Radiol 2011; 84 (997): 70-7.

9. Andreassi MG, Cioppa A, Botto N, Joksic GMS, Federici C, Ostojic M, et al. Somatic DNA damage in interventional cardiologists: a case-control study. Faseb J 2005; 17 (3): 1-17.

10. Zakeri F, Hirobe T, Akbari Noghabi K. Biological effects of low-dose ionizing radiation exposure on interventional cardiologists. Occup Med (Lond). 2010 Sep; 60.

11. Servomaa A, Karppinen J. The dose-area product and assessment of the occupational dose in interventional radiology. Radiat Prot Dosimetry [Internet]. 2001; 96 (1-3): 235-6. Available from: http://www.ncbi.nlm.nih. gov/pubmed/11586738.

12. Zett-Lobos C, Vera-Muñoz F, Arriola-Álvarez K, Díaz-Ramos O, Gamarra J, Fernández-Palomo C, et al. ¿Es suficiente la protección otorgada por gafas plomadas en cardiología intervencionista? Rev Med Chile 2013; 141: 63-9.

13. Torres HJ, Merello L, Ramos SA, Aninat MA, Becerra LE, Mora AD, et al. Prevalence of cardiac allograft vasculopathy assessed with coronary angiography versus coronary vascular ultrasound and virtual histology. Transplant Proc. 43 (6): 2318-21.

14. Ingwersen M, Drabik A, Kulka U, Oestreicher U, Fricke $\mathrm{S}$, Krankenberg H, et al. Physicians' radiation exposure in the catheterization lab: does the type of procedure matter? JACC Cardiovasc Interv 2013; 6 (10): 1095-102.

15. Le Heron J, Padovani R, Smith I, Czarwinski R. Radiation protection of medical staff. Eur J Radiol. Elsevier Ireland Ltd 2010; 76 (1): 20-3. 